\title{
Clinical and neuroradiological differences of paediatric acute disseminating encephalomyelitis with and without antibodies to the myelin oligodendrocyte glycoprotein
}

\author{
M Baumann, ${ }^{1} \mathrm{~K}$ Sahin, ${ }^{1} \mathrm{C}$ Lechner, ${ }^{1} \mathrm{E}$ M Hennes, ${ }^{1,2} \mathrm{~K}$ Schanda, ${ }^{3} \mathrm{~S} \mathrm{Mader}^{3,4}$ \\ M Karenfort, ${ }^{5}$ C Selch, ${ }^{6} \mathrm{M} \mathrm{Häusler,}^{7}$ A Eisenkölbl, ${ }^{8} \mathrm{M}$ Salandin, ${ }^{9}$ \\ U Gruber-Sedlmayr, ${ }^{10}$ A Blaschek ${ }^{11}{ }^{11}$ Kraus, $^{12} \mathrm{~S} \mathrm{Leiz,}^{13}$ J Finsterwalder, $^{14}$

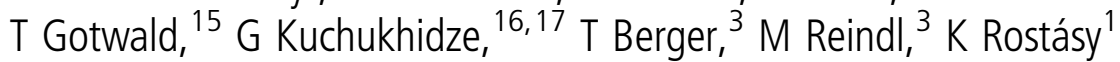

\begin{abstract}
- Additional material is published online only. To view please visit the journal online (http://dx.doi.org/10.1136/ jnnp-2014-308346).

For numbered affiliations see end of article.
\end{abstract}

\section{Correspondence to} Professor Kevin Rostásy, Division of Pediatric Neurology, Department of Pediatrics I, Medical University Innsbruck, Anichstrasse 35, Innsbruck A-6020, Austria; Kevin.Rostasy@uki.at

Received 21 April 2014 Revised 3 July 2014 Accepted 19 July 2014 Published Online First 13 August 2014

\section{SLinked}

- http://dx.doi.org/10.1136/ jnnp-2014-308723

- http://dx.doi.org/10.1136/ jnnp-2014-309057

\section{CrossMark}

To cite: Baumann $\mathrm{M}$, Sahin $\mathrm{K}$, Lechner $\mathrm{C}$, et al. $J$ Neurol Neurosurg Psychiatry 2015;86: 265-272.

\section{ABSTRACT}

Background Myelin oligodendrocyte glycoprotein (MOG) antibodies have been recently described in children with acute disseminating encephalomyelitis (ADEM), but the clinical and neuroradiological characterisation of this subgroup is lacking.

Objective To compare the clinical and neuroradiological features of paediatric ADEM with and without MOG antibodies.

Methods Clinical course, cerebrospinal fluid (CSF)-, MRI studies, outcome and MOG status of 33 paediatric ADEM prospectively studied were reviewed.

Results MOG antibodies (median 1:2560; range 1:160-1:20 480) were detected in 19 children with ADEM. The majority of children showed a decline of serum MOG-IgG titres over time. Children with MOG antibodies did not differ in their age at presentation, sex ratio, the presence of oligoclonal bands, clinical symptoms or initial severity, apart from a higher CSF cell count $(p=0.038)$, compared with children without MOG antibodies. In addition, further relapsing demyelinating episodes associated with MOG antibodies were observed only in children with MOG antibodies. All 19 children with MOG antibodies had a uniform MRI pattern, characterised by large, hazy and bilateral lesions and the absence of atypical MRI features (eg, mainly small lesions, well-defined lesions), which was significantly different compared to that of children without MOG antibodies ( $p=0.003$; and $p=0.032$, respectively). In addition, children with MOG antibodies had involvement of more anatomical areas ( $p=0.035)$ including the myelon characterised by a longitudinally extensive transverse myelitis ( $p=0.003)$, more often a complete resolution of lesions $(p=0.036)$ and a better outcome ( $p=0.038)$.

Conclusions Patients with ADEM with MOG antibodies in our cohort had a uniform MRI characterised by large, bilateral and widespread lesions with an increased frequency of longitudinal extensive transverse myelitis and a favourable clinical outcome in contrast to children lacking MOG antibodies.

\section{INTRODUCTION}

Acute disseminated encephalomyelitis (ADEM) syndrome is characterised by a clinical polyfocal event of the central nervous system (CNS) with an encephalopathy ranging from behavioural change to alteration in consciousness. ${ }^{1}$ Brain MRI usually shows diffuse, poorly demarcated and bilateral lesions involving most predominantly the cerebral white matter and spine. ${ }^{2}$ ADEM commonly follows a monophasic disease course and is associated with a good prognosis. However, a small subset of children with ADEM can have more than one event. ${ }^{1}$ Relapsing disease following ADEM indicates a chronic disorder and most often leads to the diagnosis of multiple sclerosis (MS) or neuromyelitis optica (NMO). ${ }^{3-6}$

Biomarkers predicting the course of the disease are lacking. Recently, it has been shown that serum IgG antibodies to myelin oligodendrocyte glycoprotein (MOG) are present in approximately up to $40 \%$ children with ADEM..$^{7-10}$ Typically, MOG antibodies drop to undetectable levels in follow-up samples of children with monophasic disease. ${ }^{78}$

Further, recent studies using cell-based immunoassays have demonstrated the presence of MOG-IgG antibodies in paediatric patients with MS, aquaporin-(AQP)-4-antibody seronegative $\mathrm{NMO}$, isolated optic neuritis (ON) or transverse myelitis (TM), but only rarely in adult patients with these disorders. ${ }^{11-16}$

We delineate the clinical and neuroradiological features and outcome of children with ADEM according to the serum MOG antibody status.

\section{METHODS}

Patients

Between 2009 and 2013, 231 children with a suspected acute demyelinating event such as ADEM, $\mathrm{ON}, \mathrm{NMO}$ or a clinically isolated syndrome (CIS) were sent to our attention from different hospitals in Germany, Austria and Italy for further testing of serum MOG-IgG and AQP4-IgG antibodies and included in an ongoing prospective study.

From this cohort, 39 paediatric patients were initially identified who fulfilled the diagnostic criteria of ADEM based on the International Paediatric MS Study Group (IPMSSG) recommendations which included a first polyfocal, clinical CNS event with a presumed inflammatory demyelinating cause, 
encephalopathy that cannot be explained by fever, systemic illness or postictal symptoms and an abnormal brain MRI during the acute phase compatible with ADEM and not indicative of another CNS disease. ${ }^{1}$ In addition, all children should have had a complete set of investigations (clinical information, cerebrospinal fluid (CSF) studies, MRI, serum sample). Six children were subsequently excluded because they were assigned an alternative diagnosis on follow-up (figure 1).

The majority of the final cohort of 33 children had a serological, immunological workup (white blood cells, antinuclear antibodies), metabolic screen (eg, serum lactate, amino acids and urinary organic acids) and CSF studies (cell count, oligoclonal bands (OCBs)) at the referring hospital. All 33 children were seen subsequently by the referring physicians. Clinical follow-up information including neurological symptoms and the presence of cognitive problems (eg, learning or attentional problems) and other sequelae (eg, epilepsy) was obtained via a standardised questionnaire in the majority of cases filled out by the referring physicians in general every 6 months -1 year.

All MRI studies were assessed including the following sequences (MB, TG, GK): T2-axial, fluid attenuated inversion recovery-axial, T2-sagittal, T1-axial with a contrast medium, myelon T2-sagittal and T1-sagittal with a contrast medium. Each MRI was then scored according to the location of lesions in five anatomical areas and assigned a score between 1 and 5 (supratentorial white matter (juxtacortical, non-juxtacortical and non-periventricular white matter, periventricular white matter and corpus callosum); thalamus/basal ganglia; brainstem; cerebellum; myelon with and without longitudinal extensive TM (LETM)) (KR, MB). ${ }^{17}$ In 3/33 children, the scores obtained by the two reviewers were different. MRIs of these children were reviewed and consensus agreement was reached.

In a second step, the configuration of lesions (blurred/hazy, large ( $>2 \mathrm{~cm}$ on the axial sequence) or contrast-enhancing) was

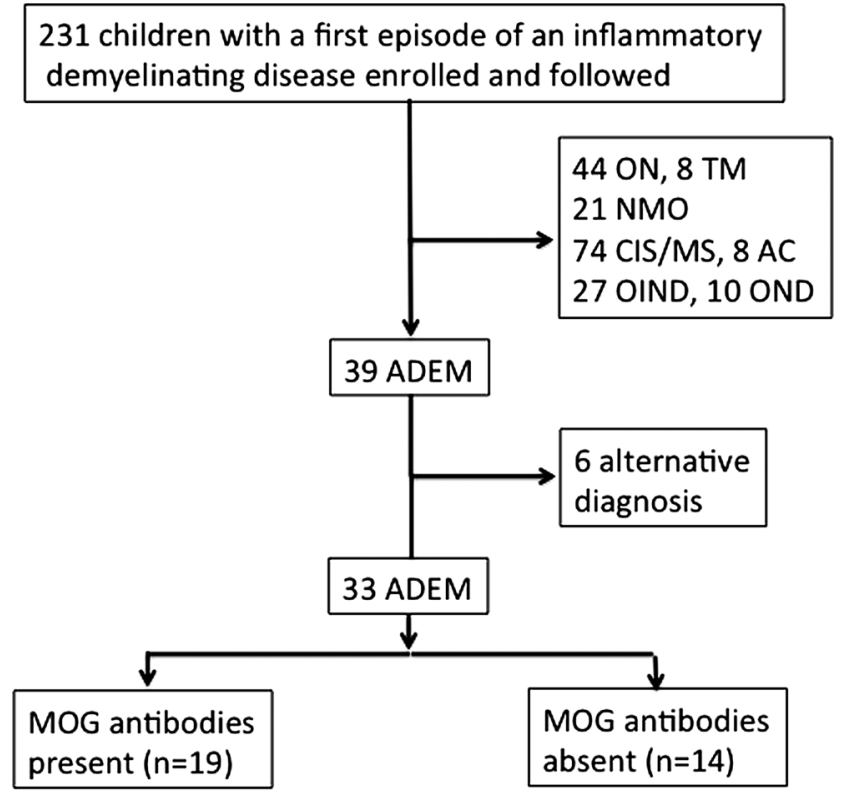

Figure 1 Flow chart Study profile. AC, acute cerebellitis; ADEM, acute disseminating encephalomyelitis, CIS, clinical isolated syndrome; MOG, myelin oligodendrocyte glycoprotein; MS, multiple sclerosis; OIND, other inflammatory neurological diseases; ON, optic neuritis; OND, other neurological diseases; NMO, neuromyelitis optica; TM, transverse myelitis. analysed and scored with yes or no $(\mathrm{KR}, \mathrm{MB})$. No differences between the reviewers were recorded.

In addition, the following MRI features were classified as atypical for ADEM: lesions only in one anatomical area, welldefined lesions, diffuse white matter involvement without clear lesional character, T1- hypointense lesions, predominantly small lesions $<2 \mathrm{~cm}$, periventricular lesions, lesions perpendicular to the corpus callosum or cortical lesions. In children who had a combination of atypical features such as well-defined lesions together with a periventricular or perpendicular location to the long axis of the corpus callosum, we additionally classified this pattern as an MS-like pattern. ${ }^{18}$ The presence of more than one atypical feature classified the MRI as atypical for ADEM. In the category atypical features, 17 features were described. In three children, the reviewers differed in their judgement. The corresponding MRIs were reviewed and consensus agreement was reached.

MRI follow-up studies were available from 31/33 children and compared in the same fashion as the initial MRIs. In order to compare the initial with the follow-up MRI results, the findings were categorised into four subgroups: complete resolution, minor residuals (few remaining T2 signal changes, but much improved), moderate residuals (only minor improvement of T2 signal changes) and marked residuals (eg, atrophy) (MB and KR).

In 29/39 children, MRI was performed on scanners with a field strength of $1.5 \mathrm{~T}$, in $7 / 39$ children with a $3.0 \mathrm{~T}$ scanner and in $3 / 39$ children with a $1.0 \mathrm{~T}$ scanner.

All serum samples were analysed for the presence of MOG-IgG and AQP4-IgG antibodies by cell-based assays as previously described and were obtained before anti-inflammatory therapy with steroids was started. ${ }^{8} 19$ Screening was performed at dilutions of $1: 20$ and 1:40 by at least two independent clinically blinded investigators (KS, SM and MR), and positive serum samples were further diluted to determine the titre levels of antibodies. Titre levels of $\geq 1: 160$ were classified as high titres as previously described. ${ }^{8}$ Serum follow-up MOG samples were tested from 25 children.

\section{Statistical analysis}

Statistical analysis was performed using IBM SPSS, release V.18.0 (IBM Corporation). We compared clinical, demographic, neuroradiological and serological data using the Kruskal-Wallis test, Mann-Whitney test, Wilcoxon rank test, Fisher exact test and $\chi^{2}$ test. Statistical significance was defined as a two-sided $\mathrm{p}$ value of less than 0.05 , and Bonferroni corrections were made for multiple comparisons when appropriate.

\section{RESULTS}

Patients

From a cohort of 231 children with a first demyelinating event, 39 patients were diagnosed with ADEM, 74 with CIS/MS, 44 paediatric patients had ON, eight had TM and 21 had NMO. ${ }^{1}$ Eight children were diagnosed with an episode of acute cerebellitis, 27 had other inflammatory neurological diseases (eg, neuroborreliosis) and 10 children had other neurological diseases (OND, eg, migraine; figure 1).

Initially, we included all 39 children diagnosed with ADEM (figure 1). Six children were subsequently excluded because they were assigned a different diagnosis on follow-up including MS $(\mathrm{n}=1)$, Guillain-Barré syndrome $(\mathrm{n}=1)$, epilepsy due to a KCNQ2 mutation $(n=1)$, CNS vasculitis $(n=2)$ and tick-borne encephalitis $(n=1)$.

The final cohort of 33 paediatric ADEM consisted of 15 girls and 18 boys with a median age of 5 years (range 1-17 years) 
and a median follow-up of 27 months (range 5-92 months). All 33 children were treated with intravenous methylprednisolone; 9 children received additional immunomodulatory treatments (see online supplementary eTable 1).

\section{Serum antibody status to MOG and AQP4}

In a first step, the presence of serum IgG antibodies to MOG and AQP4 were evaluated with a live cell staining immunofluorescence assay, as previously described. ${ }^{8} 1920$ All 33 children were tested negative for AQP4-IgG antibodies (data not shown). In 19/33 (58\%) patients with ADEM, serum MOG-IgG antibodies of $\geq 1: 160$ (high titre) were detected (median 1:5120, range 1:160-1:20 480; table 1). No child from the MOG negative group had detectable MOG antibodies in the lower range (1:20-1:80). Seventeen out of 19 children had a titre of $1: 1280$ or higher, whereas only 2/19 children had MOG-IgG titres of $1: 160$. At the last follow-up, $8 / 18$ children were still positive for MOG-IgG antibodies and 16/18 children showed a decline of $>2$ titre levels of serum MOG-IgG titres over time (median MOG titre at last follow-up 1:80, range: $0-1: 5120)$. There were no significant differences in the follow-up time between patients who were MOG-IgG positive (median 30, range 9-72 months) or MOG-IgG negative (median 27, range 5-92 months) at last follow-up.

To further analyse if children with $(n=19)$ and without $(n=14)$ serum MOG-IgG antibodies revealed any differences, we compared both subgroups (table 1).

\section{Demographic and clinical data}

Children with or without MOG antibodies did not differ in their age, sex ratio and range of clinical symptoms, apart from a higher number of CSF white blood cells and, less often, emotional or behavioural problems at initial presentation $(p=0.038$ and $\mathrm{p}=0.026$, respectively) (table 1 ).

Interestingly, 4/19 children with MOG antibodies continued to have further demyelinating episodes. All episodes occurred between 1 month and 3 years after the initial event. Three children had up to two episodes of ON (Pat.16 (2 episodes of ON 2 and 3 years later); Pat.18 (1 episode of ON 2 months later); Pat.19 (1 episode of ON 2 years later)) that were subsequently classified as acute disseminating encephalomyelitis followed by ON (ADEMON) as described previously. ${ }^{20}$ One child in this subgroup had, in addition, two further episodes reminiscent of ADEM (Pat.16). One child (Pat.17) experienced a second episode of ADEM 3 month later that was classified as MDEM (multiphasic acute disseminating encephalomyelitis; see online supplementary eTable 1).

Follow-up antibody titres from up to six different time points (19-61 months) were available from all four children. MOG antibody titres remained high in all children apart from one child with ADEMON (Pat.18), although it was lower as compared to the initial titre. There was no difference in the magnitude of the initial MOG antibody level in children with only one or more than one episode.

\section{Neuroimaging}

First, the presence of lesions in the initial brain and spinal MRI was scored in five anatomical areas in addition to the configuration of lesions.

All 19 children with ADEM and MOG antibodies had a uniform MRI pattern, characterised by large, hazy and bilateral lesions, and in the large majority of children, no atypical MRI features, which was highly significant compared with children without MOG antibodies $(\mathrm{p}=0.003$; figure 2$)$. In addition, children with MOG antibodies had involvement of more anatomical areas $(p=0.035)$ and, in particular, the myelon $(p=0.003)$. In 14/19 children with MOG antibodies, a spinal MRI was performed, of whom 13 children had LETM (92.9\%), which was also highly significant in contrast to children without MOG antibodies, who only showed an LETM in 4/12 spinal MRIs. Eight of 13 children with LETM and MOG antibodies suffered from spinal symptoms, in contrast to children with absent MOG antibodies and LETM $(n=4)$, all of whom had spinal symptoms.

Eight of 14 children without MOG antibodies also had an MRI with large, hazy and bilateral lesions (figure 3A). Six of 14 children with ADEM and absent MOG antibodies had, in addition, two or more atypical MRI features. Well-defined lesions were present in $5 / 6$ children and combined with perpendicular or periventricular lesion in 2 children (MS-like pattern; figure 3D-H). Diffuse white matter involvement was detected in $1 / 6$ children (figure $3 \mathrm{~K}$ ) and predominantly small lesions in $2 / 6$ children (figure 3J). Further atypical features included cortical lesions $(n=1)$ (figure $3 \mathrm{~L})$, T1-hypointense lesions $(n=1)$ or lesions confined to only one anatomical region $(n=3)$ (figure 3I). Only five patients showed contrast medium enhancement in selected lesions in the inital MRI studies, revealing no differences between MOG positive or negative children.

Follow-up brain MRI was available from 17/19 children with ADEM and MOG antibodies. Spinal cord imaging was performed in 14/19 children. Ten children had a complete resolution of white matter signal changes and four children continued to show minor residual focal T2 lesions that were significant compared with children without MOG antibodies $(\mathrm{p}<0.001)$. Interestingly, $2 / 19$ children with ADEM and MOG antibodies had a follow-up study within the first month showing already a dramatic improvement. Only 3/19 children with ADEM typical MRI continued to have moderate or marked residual findings such as persisting T2 lesions or atrophy.

In the group of 14 children without MOG antibodies, only 3 children-all without atypical MRI features-had a complete resolution of signal changes. Eight of 14 children continued to have moderate to marked residual MRI findings (figure 3F; table 1). Four of these eight children with marked residual MRI findings also had severe clinical residuals. Interestingly, one child with ADEM and MOG antibodies, who subsequently developed further ADEM episodes, also showed marked residual findings on MRI such as generalised atrophy combined with a severe therapy refractory seizure disorder.

\section{Outcome}

Clinical outcome was subdivided in normal (no neurological symptoms), minor (mild neurological symptoms (eg, bladder dysfunction) or moderate/severe (persistent significant neurological symptoms or sequelae such as epilepsy, learning difficulties). Clinical recovery was significantly better in children presenting with MOG-IgG antibodies than in seronegative children $(p=0.038)$ (table 1 , see online supplementary eTable1). Mild neurological sequelae were recorded only in 3/19 children with MOG antibodies including mild bladder dysfunction and mild spastic paraplegia. One child had a temporary seizure disorder, which is currently not treated. Only one child who had further demyelinating antibodies developed a therapy-resistant seizure disorder and was classified as a severe outcome.

Nine of 14 children with ADEM and absent MOG antibodies had a normal outcome. However, 5/14 children continued to have significant sequelae ranging from epilepsy, ataxia, marked attention and learning difficulties to cognitive dysfunction (see online supplementary eTable 1$)$. 


\section{Neuro-inflammation}

Table 1 Comparison of demographic, clinical features, outcome and MRI of 33 paediatric patients with ADEM with or without MOG-IgG antibodies

\begin{tabular}{|c|c|c|c|}
\hline & \multicolumn{2}{|c|}{ MOG-IgG at baseline } & \multirow[b]{2}{*}{$p$ Value } \\
\hline & negative ( $n=14$ ) & positive $(n=19)$ & \\
\hline MOG-IgG titre (1:) at baseline & $0(0)$ & $5120(160-20480)$ & \\
\hline MOG-IgG at follow-up & $0 / 6(0.0 \%)$ & $8 / 18(44.4 \%)$ & \\
\hline MOG-IgG titre (1:) at follow-up & $0(0)$ & $80(0-5120)$ & \\
\hline Decrease of MOG-IgG titre $\geq 2$ steps & & $16 / 18(88.9 \%)$ & \\
\hline CSF OCBS & $1(7.1 \%)$ & $1 / 18(5.6 \%)$ & 1.000 \\
\hline CSF cell count $/ \mu \mathrm{L}$ & $10(1-95)$ & $53(2-339)$ & 0.038 \\
\hline Females & $6(40.0 \%)$ & $9(50.0 \%)$ & 1.000 \\
\hline Age at onset (years) & $7(1-13)$ & $4(1-17)$ & 0.084 \\
\hline \multicolumn{4}{|l|}{ Symptoms } \\
\hline Altered consciousness & $13(92.9 \%)$ & $17(89.5 \%)$ & 1.000 \\
\hline Emotional/behaviour & $6(42.9 \%)$ & $1(5.3 \%)$ & 0.026 \\
\hline Seizures & $2(14.3 \%)$ & $2(10.5 \%)$ & 1.000 \\
\hline Optic neuritis & $2(14.3 \%)$ & $3(15.8 \%)$ & 1.000 \\
\hline CN (III-XII) & $6(42.9 \%)$ & $7(36.8 \%)$ & 1.000 \\
\hline Pyramidal signs & $3(21.4 \%)$ & $3(15.8 \%)$ & 1.000 \\
\hline Ataxia & $5(35.7 \%)$ & $10(52.6 \%)$ & 0.482 \\
\hline Sensory symptoms & $2(14.3 \%)$ & $1(5.3 \%)$ & 0.561 \\
\hline Spinal symptoms & $6(42.9 \%)$ & $10(52.6 \%)$ & 0.728 \\
\hline TM (symptom+MRI lesion) & $4(28.6 \%)$ & $10(52.6 \%)$ & 0.286 \\
\hline Follow-up (months) & $25(6-61)$ & $27(5-92)$ & 1.000 \\
\hline Multiphasic disease course & $0(0.0 \%)$ & $4(21.1 \%)$ & 0.119 \\
\hline \multicolumn{4}{|l|}{ Clinical recovery } \\
\hline Complete & $9(64.3 \%)$ & $15(78.9 \%)$ & 0.038 \\
\hline Minor residuals & $0(0.0 \%)$ & $3(15.8 \%)$ & \\
\hline Severe residuals & $5(35.7 \%)$ & $1(5.3 \%)$ & \\
\hline \multicolumn{4}{|l|}{ MRI areas affected } \\
\hline 1. Supratentoriell WM & $13(92.9 \%)$ & $16(84.2 \%)$ & 1.000 \\
\hline 2. Thalamus/basal ganglia & $9(64.3 \%)$ & $17(89.5 \%)$ & 0.238 \\
\hline 3. Brainstem & $8(57.1 \%)$ & $14(73.7 \%)$ & 0.459 \\
\hline 4. Cerebellar & $5(35.7 \%)$ & $12(63.2 \%)$ & 0.166 \\
\hline 5. Spinal & $4 / 12(33.3 \%)$ & $13 / 14(92.9 \%)$ & 0.003 \\
\hline Widespread score (items 1-5) & $3(1-5)$ & $4(2-5)$ & 0.035 \\
\hline Brainstem/spinal lesions combined & $8(57.1 \%)$ & $18(94.7 \%)$ & 0.026 \\
\hline LETM & $4 / 12(33.3 \%)$ & $13 / 14(92.9 \%)$ & 0.003 \\
\hline Bilateral lesions & $14(100.0 \%)$ & $19(100.0 \%)$ & 1.000 \\
\hline Large lesions $>2 \mathrm{~cm}$ axial & $13(92.9 \%)$ & $19(100.0 \%)$ & 0.424 \\
\hline \multicolumn{4}{|l|}{ Atypical MRI features } \\
\hline Only one MRI area affected & $3(21.4 \%)$ & $0(0 \%)$ & 0.067 \\
\hline Diffuse WM involvement & $1(7.1 \%)$ & $0(0.0 \%)$ & 0.424 \\
\hline Small lesions & $2(14.3 \%)$ & $0(0.0 \%)$ & 0.090 \\
\hline T1-hypointense lesions & $1(7.1 \%)$ & $0(0.0 \%)$ & 0.424 \\
\hline Well defined borders & $5(35.7 \%)$ & $0(0.0 \%)$ & 0.008 \\
\hline Perpendicular & $1(7.1 \%)$ & $0(0.0 \%)$ & 0.424 \\
\hline Periventricular & $1(7.1 \%)$ & $1(5.3 \%)$ & 1.000 \\
\hline Cortical lesions & $1(7.1 \%)$ & $1(5.3 \%)$ & 1.000 \\
\hline Number of atypical features & $1(1-3)$ & $0(0-1)$ & 0.032 \\
\hline ADEM with $\leq 1$ atypical MRI feature & $8(57.1 \%)$ & $19(100.0 \%)$ & 0.003 \\
\hline MRI outcome available from & $14 / 14$ & $17 / 19$ & \\
\hline 1. Complete resolution & $3(21.4 \%)$ & $10(58.8 \%)$ & 0.036 \\
\hline 2. Minor residuals & $3(21.4 \%)$ & $4(23.5 \%)$ & \\
\hline 3. Moderate residuals & $1(7.1 \%)$ & $2(11.8 \%)$ & \\
\hline 4. Marked residuals & $7(50.0 \%)$ & $1(5.9 \%)$ & \\
\hline
\end{tabular}

ADEM, acute disseminating encephalomyelitis; CN, cranial nerves; CSF, cerebrospinal fluid; LETM, longitudinal extensive transverse myelitis; MOG, myelin oligodendrocyte glycoprotein; OCBs, oligoclonal bands; TM, transverse myelitis; WM, white matter. 

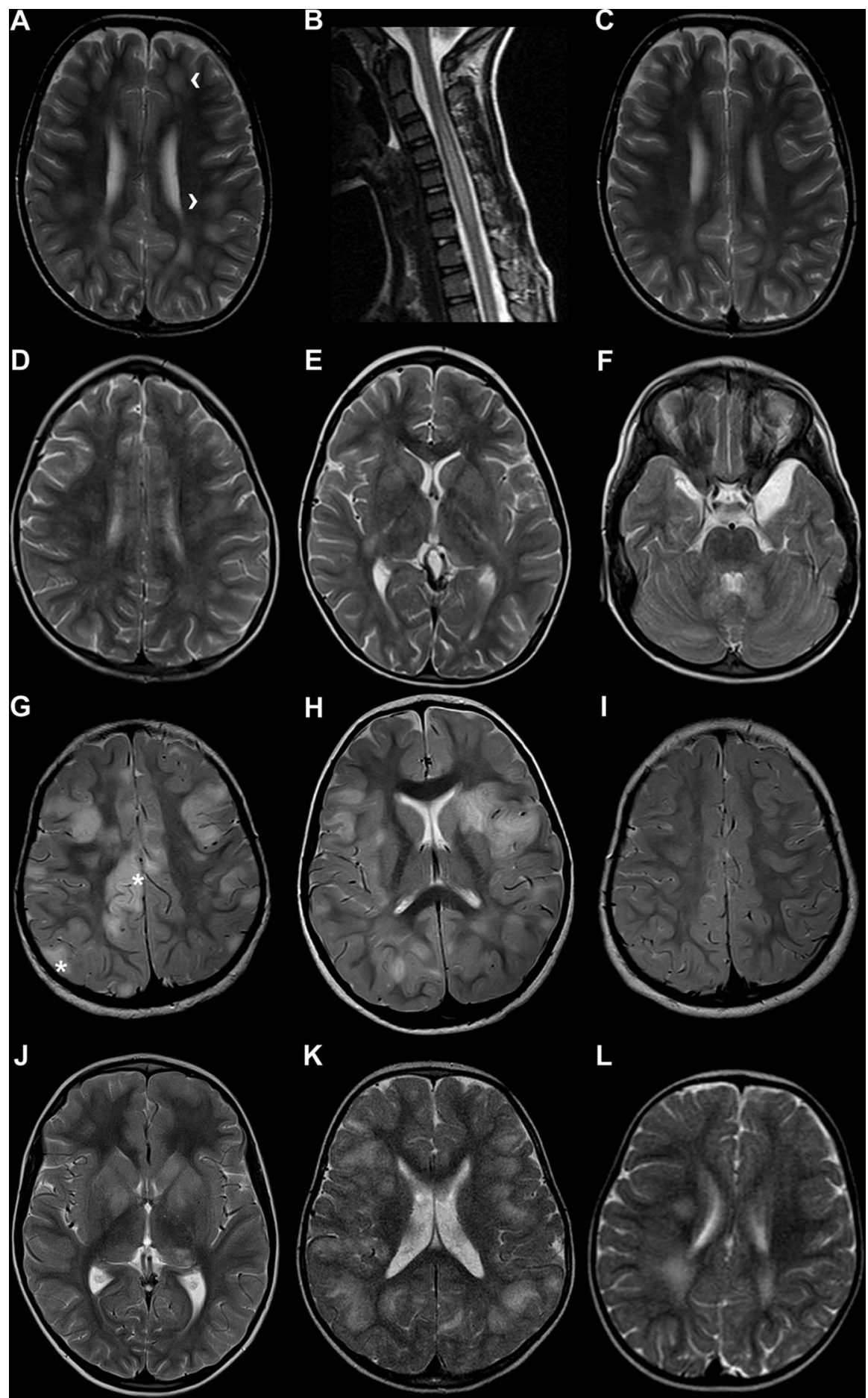

Figure 2 Imaging of 6 paediatric patients with ADEM (acute disseminating encephalomyelitis) and MOG antibodies. (A-C) Cerebral and spinal MRI of a 6-year-old patient (Pat.2) with MOG antibodies, who presented with headache, lethargy, lower limbs weakness, paraesthesia of the left arm and leg combined with urinary and bowel incontinence, revealed large and blurred lesions (arrowheads) involving both hemispheres ( $A$, axial-T2) and the spinal cord with a longitudinal extensive lesion (LETM) (B, sagittal-T2). Two weeks later, the supratentorial and spinal lesions had mostly resolved (C, axial-T2). (D-F) Cerebral MRI of a 4-year-old patient (Pat.13) with MOG antibodies, who presented with extreme lethargy, paraparesis, a thalamic pain syndrome and headache, showed widespread and hazy lesions involving both hemispheres (D and E), the thalamus, basal ganglia (E), brainstem, cerebellum ( $F$, all axial-T2) and spinal cord with LETM (not shown). (G-I) Cerebral MRI of a 2-year-old patient (Pat.9) with MOG antibodies, who presented with somnolence, opisthotonus, swallowing and respiratory problems, showed several large, blurredwhite matter and cortical lesions (asterisks, atypical feature) (G,H, all axial-T2). 5 months later, the lesions had almost disappeared (I, axial T2). (J) Cerebral MRI of a 5 year old patient (Pat.19) with MOG antibodies, who presented with headache, ataxia and lethargy, showed large and blurred lesions involving the thalamus, basal ganglia ( $\mathrm{J}$, axial-T2), brainstem, cerebellum and spinal cord with LETM (not shown). (K) Cerebral MRI of a 3-year-old patient (Pat.6) with MOG antibodies, who presented with fever, headache, dysarthria, gait disorder and right hemiparesis, showed large and blurred lesions involving both hemispheres (K, axial-T2), the thalamus, basal ganglia, brainstem and spinal cord with LETM (not shown). (L) Cerebral MRI of a 1-year-old patient (Pat.4) with MOG antibodies, who presented with ataxia, a tendency to fall, strabismus convergens and lethargy, showed large and blurred lesions involving both hemispheres ( $\mathrm{L}$, axial-T2), the thalamus and cerebellum (not shown). 

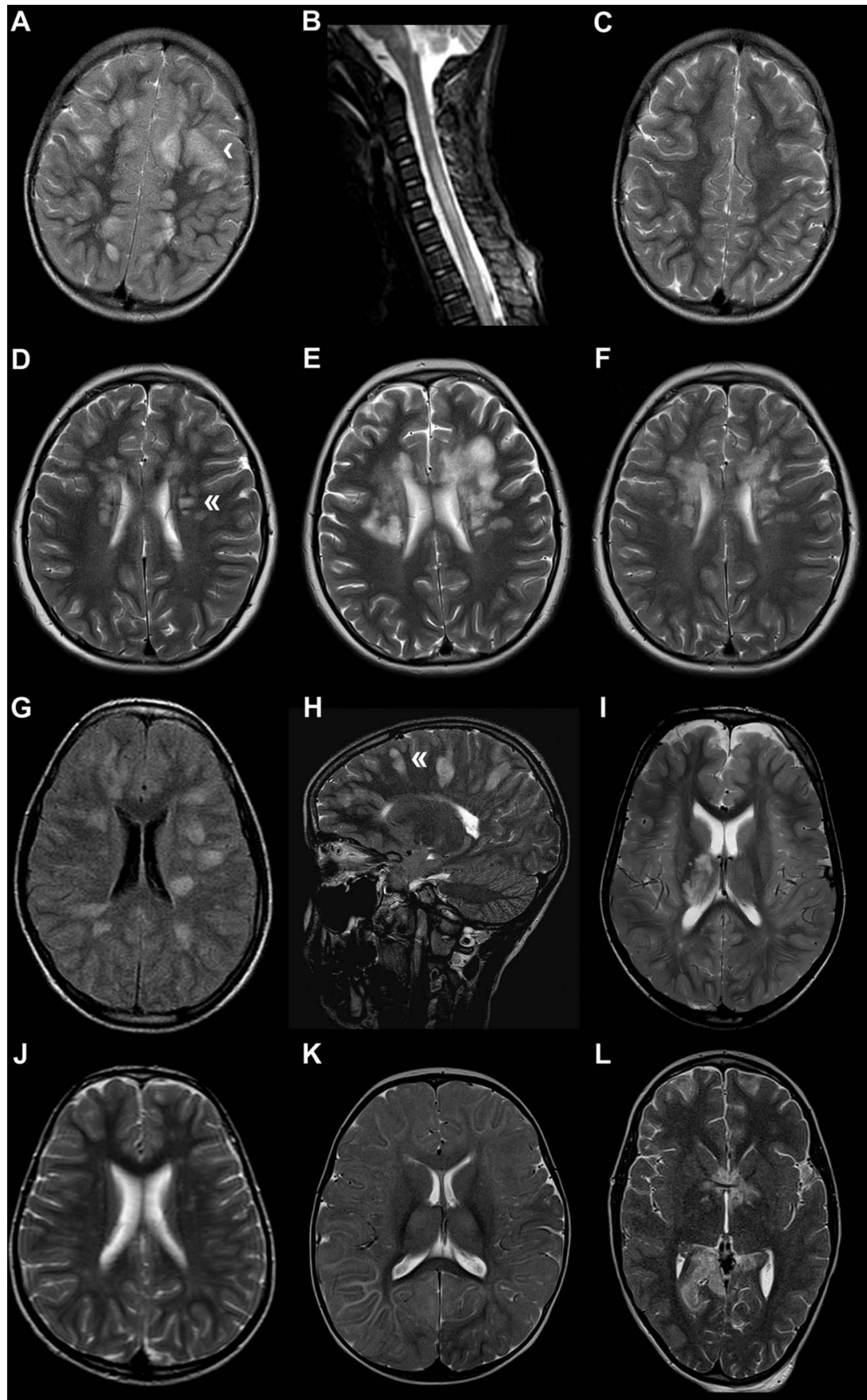

K

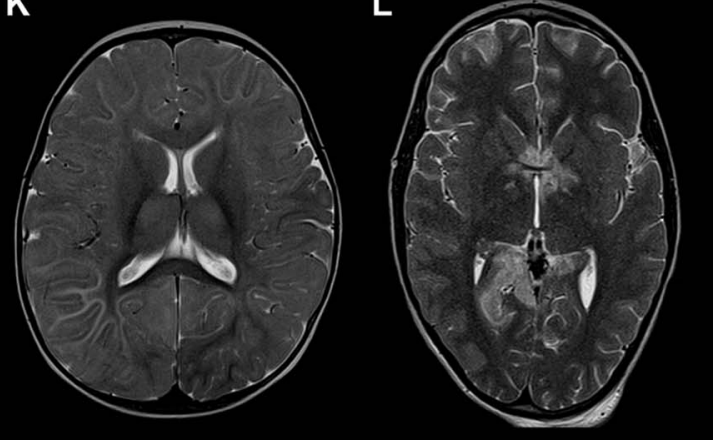

Figure 3 Imaging of 7 paediatric patients with acute disseminating encephalomyelitis (ADEM) lacking MOG antibodies. (A-C) MRI of a 5-year-old patient (Pat.21) with ADEM but without MOG antibodies revealed a MR-pattern reminiscent of children with ADEM and MOG antibodies with widespread, blurred (arrowhead), large lesions involving both hemispheres ( $A$, axial-T2), the thalamus and brainstem (not shown), and longitudinally extensive transverse myelitis (LETM) (B, sagittal-T2). Three months later, the lesions had mostly resolved (C, axial-T2). (D-F) MRI of a patient (Pat.31) lacking MOG antibodies who presented at the age of 11 years with aggressive behaviour, right haemiparesis and finally marked somnolence showed mainly well-defined and periventricular (double arrowhead) MS (multiple sclerosis)-like lesions which classified as an atypical feature (D). Cerebral MRI obtained one month later revealed extension of the previously noted lesions (E) which again decreased in size and signal intensity three months later ( $\mathrm{F}$, all axial-T2). (G and H) Cerebral MRI of a 13-year-old patient (Pat.29) with ADEM and absent MOG antibodies, who presented with symptoms including behavioural changes, bilateral motor weakness and ataxia, also showed large perpendicular lesions with mostly well-defined borders (double arrowhead) and was classified as an MS like pattern (G, axial fluid attenuated inversion recovery; $H$, saggital-T2). The patient had no further clinical episodes and also no new lesion formation. (I) Cerebral MRI of a 7-year-old patient (Pat.33) with ADEM lacking MOG antibodies, who presented with symptoms including mental changes and a tetraparesis, was characterised by bilateral lesions mainly confined to the basal ganglia with partly well-defined borders (I, axial-T2). (J) Cerebral MRI of a 4-year-old patient (Pat. 28) diagnosed with ADEM lacking MOG antibodies, who presented with somnolence, aggression, urinary and bowel incontinence combined with ataxia and visual problems, revealed predominantly small lesions in the white matter ( $\mathrm{J}$, axial-T2), which did not change over time (not shown). (K) Cerebral MRI of a 1-year-old patient (Pat.30) with ADEM and absent MOG antibodies, who presented with fever, somnolence and focal seizures, showed diffuse white matter involvement without clear lesions (K, axial-T2). (L) Cerebral MRI of a 9-year-old patient (Pat.32) with ADEM lacking MOG antibodies, who presented with severe headache, internuclear ophthalmoplegia, right haemiparesis, bladder dysfunction and stupor, showed mostly well-defined lesions involving white matter, the basal ganglia, cortex (L, axial-T2), brainstem and cerebellum (not shown). MOG, myelin oligodendrocyte glycoprotein. 


\section{DISCUSSION}

We describe the clinical and neuroradiological features of paediatric patients with ADEM with serum MOG antibodies, which have been recently detected in a group of children and adults with ADEM, ON and NMO spectrum disorders. ${ }^{7-15}$

In our cohort of 33 paediatric patients with ADEM, serum MOG antibodies were detected in 19 children. From these 19 MOG IgG seropositive children, 15 had a monophasic disease course and 4 continued to have further episodes. All children with MOG antibody positive monophasic ADEM showed declining antibody levels (available from 14 patients) over the course of months to years, which is in line with previous reports. ${ }^{7}$ All children with further demyelinating episodes (ON, MDEM) had high MOG antibody levels, which still remain high in $3 / 4$ children even after a follow-up period of 61 months as described previously. ${ }^{20}$

All children in our cohort had initially an encephalopathy combined with focal neurological signs and an MRI compatible with ADEM.

Comparison of the demographic data and initial clinical features based on the MOG antibody status did not reveal any difference apart from an elevated CSF cell count and, less often, behavioural/ emotional problems in children with MOG antibodies.

However, important differences were noted on MRI and clinical outcome. Analysis of the initial MRI studies revealed a nearly uniform pattern in all 19 children with ADEM and MOG antibodies characterised by hazy, bilateral lesions without clear boundaries, the absence of two or more atypical MRI features and a more widespread distribution of lesions. In particular, the myelon was more often affected in children with MOG antibodies with lesions spanning more than three segments (LETM). Similar observations were recently made by others who found that adults with an NMO spectrum disorder and MOG antibodies also have frequently long lesions extending even into the lumbar spinal cord. ${ }^{212}$

Another important finding was that in the majority of children with MOG antibodies, signal changes were transient and resolved in follow-up studies. On the other hand, in children lacking MOG antibodies, only three children had a complete resolution of signal changes and more than half of all children without MOG antibodies continued to have moderate to marked residual MRI findings. The transient nature of MRI findings has been previously noted in individual patients with autoimmune diseases and MOG antibodies. ${ }^{11}$ Interestingly, Saadoun et $\mathrm{al}^{23}$ recently showed in a mouse model that intraparenchymal injected MOG antibodies induced lesions, which were transient and not associated with complement activation or recruitment of inflammatory cells in the rodent brain.

The spectrum of MRI findings in children with ADEM and absent MOG antibodies was less homogen and showed a wider range of findings. One side of the spectrum included children who had an MRI with large, bilateral and widespread lesions but absent MOG antibodies. The other side of the spectrum consisted of children who had absent MOG antibodies and an MRI pattern with predominantly atypical features indicating that a diagnosis other than ADEM should be entertained. Initially, we had included 39 children who fulfilled the diagnostic criteria of ADEM. However, in the follow-up period, six children were assigned a different diagnosis. Interestingly, all six children were negative for MOG antibodies and 5/6 children had two or more atypical MRI features and no resolution of lesions on follow-up. The fact that a substantial number of children eventually will have another diagnosis has already been pointed out by others, indicating the need for biomarkers that help to predict the eventual course of the disease. ${ }^{3}$

Our findings have important clinical implications. Children with ADEM who are seropositive for MOG antibodies and have a typical MRI pattern form a distinct group of paediatric patients associated with a good outcome. Further, we identified a subgroup of MOG IgG negative children with an MRI pattern, indistinguishable from MOG positive patients with ADEM, indicating that other autoantibodies directed against other antigens of the myelin sheath or glial components might be involved in the disease process. Lastly, other diagnoses should be sought in children with ADEM who have absent MOG antibodies and atypical MRI features.

Several limitations should be addressed. Although the majority of MRI studies were performed on a $1.5 \mathrm{~T}$ scanner, MRI was performed in 10 children on scanners with a different field strength, leading potentially to a different quality of images and subsequent results. In addition, as detailed in previous studies, children with ADEM and MOG antibodies can have subsequent episodes of ON. ${ }^{20}{ }^{24}$ Therefore, it would have been important to assess if children with an initial episode of ADEM and MOG antibodies also have involvement of the optic nerve. Unfortunately, the MRI protocols did not explicitly include sequences to evaluate the optic nerve.

\section{CONCLUSIONS}

Our study shows that patients with ADEM with MOG antibodies had a uniform MRI characterised by large, bilateral and widespread lesions with an increased frequency of LETM and a favourable clinical outcome including the children with further demyelinating events in contrast to children lacking MOG antibodies.

\section{Author affiliations}

${ }^{1}$ Division of Pediatric Neurology, Department of Pediatrics I, Innsbruck Medical University, Innsbruck, Austria

${ }^{2}$ Clinical Department of Neurology, Klinikum Bogenhausen, Munich, Germany

${ }^{3}$ Clinical Department of Neurology, Innsbruck Medical University, Innsbruck, Austria

${ }^{4}$ The Feinstein Institute for Medical Research, Center for Autoimmune and

Musculoskeletal Diseases, Manhasset, New York, USA

${ }^{5}$ Department of General Pediatrics, Pediatric Neurology, Heinrich Heine University

Düsseldorf, Düsseldorf, Germany

${ }^{6}$ Behandlungszentrum Vogtareuth, Vogtareuth, Germany

${ }^{7}$ Department of Pediatrics, Division of Neuropediatrics and Social Pediatrics,

University Hospital, Aachen, Germany

${ }^{8}$ Department of Pediatrics, Landes- Frauen- und Kinderklinik Linz, Linz, Austria

${ }^{9}$ Department of Pediatrics, Bozen Hospital, Bozen, Italy

${ }^{10}$ Department of Pediatrics, Graz Medical University, Graz, Austria

${ }^{11}$ Department of Pediatric Neurology and Developmental Medicine, Dr von Hauner Children's Hospital, University of Munich, Munich, Germany

${ }^{12}$ Department of Pediatrics, Klinikum rechts der Isar, Technische Universität, Munich, Germany

${ }^{13}$ Pediatric Neurology, Department of Pediatrics, Klinikum Dritter Orden, Munich, Germany

${ }^{14}$ Division of Pediatric Neurology, Department of Pediatrics, Klinikum Mutterhaus der Borromäerinnen, Trier, Germany

${ }^{15}$ Radiological Institute, Kettenbrücke, Innsbruck, Austria

${ }^{16}$ Department of Neuroradiology, Medical University of Innsbruck, Innsbruck, Austria

${ }^{17}$ Department of Neurology, Paracelsus Medical University of Salzburg, Salzburg, Austria

Acknowledgements Kevin Rostásy and Markus Reindl had full access to all of the data in the study and take responsibility for the integrity of the data and the accuracy of the data analysis.

Contributors MB: design, data acquisition, revising the manuscript, final approval. $\mathrm{KS}$ : data acquisition, revising the manuscript, final approval. $\mathrm{CL}, \mathrm{JF}, \mathrm{EMH}, \mathrm{KS}, \mathrm{SM}$, MK, CS, AE, UG-S, VK, MH, AB, MS and SL were involved in the data acquisition, revising the manuscript, final approval. TG: data acquisition, analysis, final approval of the version to be published. GK: data acquisition, analysis, final approval of the version to be published. TB, KR and MR were involved in the design, data acquisition, analysis, final approval of the version to be published. 
Funding This study was supported by research grant No.14158 (KR) of the Jubilaeumsfonds of the Austrian National Bank.

\section{Competing interests None.}

Ethics approval The study was approved by the Ethics Committee of the Medical Faculty of the University of Innsbruck, Austria (Study number AM4059).

Provenance and peer review Not commissioned; externally peer reviewed.

\section{REFERENCES}

1 Krupp LB, Tardieu M, Banwell B, et al. International Pediatric MS Study Group criteria for pediatric multiple sclerosis and immune-mediated central nervous system demyelinating disorders: revisions to the 2007 definitions. Mult Scler 2013;19:1261-7.

2 Rostásy K, Nagl A, Lütjen $\mathrm{S}$, et al. Clinical outcome of children presenting with a severe manifestation of acute disseminated encephalomyelitis. Neuropediatrics 2009;40:211-17.

3 Mikaeloff Y, Caridade G, Husson B, et al. Acute disseminated encephalomyelitis cohort study: prognostic factors for relapse. Eur J Paediatr Neurol 2007;11:90-5.

4 Banwell B, Shroff M, Ness JM, et al. MRI features of pediatric multiple sclerosis. Neurology 2007;68(16 Suppl 2):46-53.

5 Alper G, Heyman R, Wang L. Multiple sclerosis and acute disseminated encephalomyelitis diagnosed in children after long-term follow-up: comparison of presenting features. Dev Med Child Neurol 2009;51:480-6.

6 Tenembaum S, Chamoles N, Fejerman N. Acute disseminated encephalomyelitis: a long-term follow-up study of 84 pediatric patients. Neurology 2002;59: 1224-31.

7 Pröbstel AK, Dornmair K, Bittner R, et al. Antibodies to MOG are transient in childhood acute disseminated encephalomyelitis. Neurology 2011;77:580-8.

8 Di Pauli F, Mader S, Rostasy K, et al. Temporal dynamics of anti-MOG antibodies in CNS demyelinating diseases. Clin Immunol 2011;138:247-54.

9 O'Connor KC, McLaughlin KA, De Jager PL, et al. Self-antigen tetramers discriminate between myelin autoantibodies to native or denatured protein. Nat Med 2007;13:211-17.

10 Brilot F, Dale RC, Selter RC, et al. Antibodies to native myelin oligodendrocyte glycoprotein in children with inflammatory demyelinating central nervous system disease. Ann Neurol 2009;66:833-42.
11 Rostasy K, Mader S, Schanda K, et al. Anti-MOG antibodies in children with optic neuritis. Arch Neurol 2012;69:752-6.

12 Rostásy K, Mader S, Hennes E, et al. Persisting myelin oligodendrocyte glycoprotein antibodies in aquaporin-antibody negative pediatric neuromyelitis optica. Mult Scler 2013;19:1052-9.

13 McLaughlin KA, Chitnis T, Newcombe J, et al. Age-dependent B cell autoimmunity to a myelin surface antigen in pediatric multiple sclerosis. J Immunol 2009;183:4067-76.

14 Kitley J, Woodhall M, Waters $\mathrm{P}$, et al. Myelin-oligodendrocyte glycoprotein antibodies in adults with a neuromyelitis optica phenotype. Neurology 2012;79:1273-7.

15 Hacohen Y, Absoud M, Woodhall M, et al. Autoantibody biomarkers in childhood-acquired demyelinating syndromes: results from anational surveillance cohort. J Neurol Neurosurg Psychiatry 2014;85:456-61.

16 Reindl M, Di Pauli F, Rostásy K, et al. The spectrum of MOG autoantibodyassociated demyelinating diseases. Nat Rev Neurol 2013;9:455-61.

17 Verhey LH, Branson HM, Shroff MM, et al.; Canadian Pediatric Demyelinating Disease Network. MRI parameters for prediction of multiple sclerosis diagnosis in children with acute CNS demyelination: a prospective national cohort study. Lancet Neurol 2011;10:1065-73.

18 Mikaeloff Y, Adamsbaum C, Husson B, et al. MRI prognostic factors for relapse after acute CNS inflammatory demyelination in childhood. Brain 2004;127:1942-7.

19 Mader S, Gredler V, Schanda K, et al. Complement activating antibodies to myelin oligodendrocyte glycoprotein in neuromyelitis optica and related disorders. J Neuroinflammation 2011;8:184

20 Huppke P, Rostasy K, Karenfurt M, et al. Acute disseminated encephalomyelitis followed by recurrent or monophasic optic neuritis in pediatric patients. Mult Scler 2013;19:941-6.

21 Sato KD, Callegaro D, Lana- Peixoto MA, et al. Distinction between MOG antibody-positive and AQP4 antibody-positive NMO spectrum disorders. Neurology 2014;82:1-8

22 Kitley J, Leitle MI, Küker W, et al. Longitudinally extensive transverse myelitis with and without aquaporin 4 antibodies. JAMA 2013;70:1375-81.

23 Saadoun S, Waters P, Owens GP, et al. Neuromyelitis optica MOG-IgG causes reversible lesions in mouse brain. Acta Neuropathol Commun 2014;2:35.

24 Miyauchi A, Monden Y, Watanabe $M$, et al. Persistent presence of the anti-myelin oligodendrocyte glycoprotein autoantibody in a pediatric case of acute disseminated encephalomyelitis followed by optic neuritis. Neuropediatrics 2014;45:e2. 


\section{Clinical and neuroradiological differences of paediatric acute disseminating encephalomyelitis with and without antibodies to the myelin oligodendrocyte glycoprotein}

M Baumann, K Sahin, C Lechner, E M Hennes, K Schanda, S Mader, M Karenfort, C Selch, M Häusler, A Eisenkölbl, M Salandin, U Gruber-Sedlmayr, A Blaschek, V Kraus, S Leiz, J Finsterwalder, T Gotwald, G Kuchukhidze, T Berger, M Reindl and K Rostásy

J Neurol Neurosurg Psychiatry 2015 86: 265-272 originally published online August 13, 2014

doi: 10.1136/jnnp-2014-308346

Updated information and services can be found at:

http://jnnp.bmj.com/content/86/3/265

\section{These include:}

Supplementary Supplementary material can be found at:

Material http://jnnp.bmj.com/content/suppl/2014/08/13/jnnp-2014-308346.DC1

References This article cites 24 articles, 3 of which you can access for free at: http://jnnp.bmj.com/content/86/3/265\#BIBL

\section{Email alerting} service

Receive free email alerts when new articles cite this article. Sign up in the box at the top right corner of the online article.

Topic Articles on similar topics can be found in the following collections Collections

\section{Notes}

To request permissions go to:

http://group.bmj.com/group/rights-licensing/permissions

To order reprints go to:

http://journals.bmj.com/cgi/reprintform

To subscribe to BMJ go to:

http://group.bmj.com/subscribe/ 